\title{
An a posteriori error estimator for model adaptivity in electrocardiology
}

\author{
L. Mirabella ${ }^{\mathrm{a}, \mathrm{b}, *}$, F. Nobile ${ }^{\mathrm{a}}$, A. Veneziani ${ }^{\mathrm{b}}$ \\ ${ }^{a}$ MOX (Modeling and Scientific Computing), Department of Mathematics, Politecnico di Milano, P.za L. da Vinci 32, 20133 Milano, Italy \\ ${ }^{\mathrm{b}}$ Department of Mathematics and Computer Science, Emory University, 400 Dowman Dr NE, 30322 Atlanta, GA, USA
}

\section{A R T I C L E I N F O}

Article history:

Received 10 August 2009

Received in revised form 15 January 2010

Accepted 8 March 2010

Available online 23 March 2010

\section{MSC:}

65M60

92C05

Keywords:

Computational electrocardiology

Model adaptivity

A posteriori error estimation

Modeling error

\begin{abstract}
A B S T R A C T
We introduce an a posteriori modeling error estimator for the effective computation of electric potential propagation in the heart. Starting from the Bidomain problem and an extended formulation of the simplified Monodomain system, we build a hybrid model, called Hybridomain, which is dynamically adapted to be either Bi- or Monodomain ones in different regions of the computational domain according to the error estimator. We show that accurate results can be obtained with the adaptive Hybridomain model with a reduced computational cost compared to the full Bidomain model. We discuss the effectivity of the estimator and the reliability of the results on simulations performed on real human left ventricle geometries retrieved from healthy subjects.
\end{abstract}

(c) 2010 Elsevier B.V. All rights reserved.

\section{Introduction}

The propagation of electrical potential in the cardiac tissue is well described by the so-called Bidomain model (see e.g. [22]), which is a system of non-linear unsteady partial differential equations coping with both the intra- and extracellular potential dynamics. Usually, the computational cost of numerical simulations of this system is high due to the degenerate parabolic nature of the model, being the time derivative vector multiplied by a singular matrix. Moreover, an accurate solution on real geometries demands for fine meshes and time steps. For these reasons, many applications consider a simplified model called Monodomain. It relies however on an assumption on the fibers conductivity which is not always verified and this model is not able to predict certain physiological and pathological patterns, especially in the neighborhood of a propagating front [13]. Moreover, the standard Monodomain model does not predict correctly the front propagation velocity [20]. See also $[7,8]$ for an "improved" Monodomain model that features a front speed closer to the Bidomain one. In this work we will refer to the standard Monodomain model as described e.g. in [7].

Recent literature has been devoted to the efficient solution of the discretized Bidomain model and, in particular, to the development of efficient preconditioners (see e.g. $[7,18,26,27,30,31])$. In

* Corresponding author at: Department of Mathematics and Computer Science, Emory University, 400 Dowman Dr NE, 30322 Atlanta, GA, USA

E-mail address: lucia@mathcs.emory.edu (L. Mirabella).
[12] an extended version of the Monodomain model has been proposed as a preconditioner for solving the Bidomain system.

In this paper we follow a different approach for simulating potential propagation in the heart. More precisely, inspired by the recent literature on modeling error estimation and adaptation (see e.g. $[3,17,19,21,28])$, we combine the Bi- and Monodomain models in a model adaptivity framework. The basic idea is to confine the (more expensive) Bidomain solution to a small part of the domain at hand, while on its most part we solve the Monodomain equation. In this way, we reduce the computational time, without significantly affecting the reliability of the numerical solution.

The crucial step in this approach is the setup of a modeling error estimator able to identify the region where it is worth solving the Bidomain system. Based on the error estimate we solve a finite element discretization of the hybrid model. We actually solve the Bidomain model on some elements while in the most part of the domain we keep on solving the Monodomain system. Numerical results presented here are carried out on a real geometry retrieved from medical images and show that the hybrid model driven by our estimator is able to capture the most important features of the potential propagation described by a full Bidomain model with a good effectivity and CPU time reduction.

The paper is organized as follows. In Section 2 we introduce the Bidomain and Monodomain systems and recall their features. We introduce the extended formulation of the Monodomain model and the Hybridomain system used for the model adaptivity. Moreover, we present the semi-discretization of these problems 
(continuous in space, discrete in time). In Section 3 we introduce two quantities providing a posteriori upper and lower bounds for the modeling error and investigate their properties. In Section 4 we describe implementation details and the algorithm for the model adaptivity.

Numerical results are presented in Section 5. We refer to a real geometry of a heart retrieved by SPECT Images (courtesy of Dr. E.V. Garcia group at Emory University). We discuss the effectivity of our strategy showing the reliability of the adaptive Hybridomain model in capturing a nontrivial potential pattern, both in a healthy and a pathological case.

\section{The Bidomain, Monodomain, and Hybridomain models}

We introduce the mathematical models commonly used for describing the propagation of the electric potential in the heart, without a detailed insight of the underlying physiology. For a complete introduction, we refer e.g. to [8,22].

Let $\Omega \subset \mathbb{R}^{3}$ be a bounded region where we investigate the cardiac potential. We do not distinguish the intra- and extracellular space, meaning that we assume that the intra- and extracellular potentials $u_{\tau}(\tau=i, e)$ are both defined in the entire domain. With $u=u_{i}-u_{e}$ we denote the transmembrane potential. Cardiac tissue is made of fibers that drive the potential propagation. With $\boldsymbol{a}_{l}$ we denote the direction along the fiber, $\boldsymbol{a}_{t}$ is orthogonal to the fiber direction and in the fiber sheet and $\boldsymbol{a}_{n}$ orthogonal to the sheet. Conductivity is different along the different directions, so we denote by $\sigma_{i}^{l}(\boldsymbol{x})\left(\right.$ resp. $\left.\sigma_{e}^{l}(\boldsymbol{x})\right)$ the intracellular (resp. extracellular) conductivity in $\boldsymbol{a}_{l}(\boldsymbol{x})$ direction at point $\boldsymbol{x} \in \Omega$, and similarly by $\sigma_{i}^{t}(\boldsymbol{x})\left(\sigma_{e}^{t}(\boldsymbol{x})\right)$ and $\sigma_{i}^{n}(\boldsymbol{x})\left(\sigma_{e}^{n}(\boldsymbol{x})\right)$ the conductivities along $\boldsymbol{a}_{t}(\boldsymbol{x})$ and $\boldsymbol{a}_{n}(\boldsymbol{x})$. Following Colli Franzone and Pavarino [7], we assume the same conductivity in both the tangential and normal direction (axial isotropy), so that the conductivity tensors read

$\mathbf{D}_{\tau}(\boldsymbol{x})=\sigma_{\tau}^{t} \mathbf{I}+\left(\sigma_{\tau}^{l}-\sigma_{\tau}^{t}\right) \boldsymbol{a}_{l}(\boldsymbol{x}) \boldsymbol{a}_{l}^{T}(\boldsymbol{x})$

for $\tau=i$, e. Moreover, we assume that $\mathbf{D}_{\tau}$ satisfies a uniform ellipticity condition in $\Omega$. The current density in each domain can be computed as $\mathbf{J}_{\tau}=-\mathbf{D}_{\tau} \nabla u_{\tau}$ for $\tau=i$,e. The net current flux between the intra- and extracellular domain is assumed to be zero as a consequence of the charge conservation in an arbitrary portion of tissue. Let us denote by $I_{m}$ the ingoing membrane current flow and by $\chi$ the ratio of membrane area per volume of tissue. We therefore get

$\nabla \cdot\left(\mathbf{D}_{i} \nabla u_{i}\right)=\chi I_{m}=-\nabla \cdot\left(\mathbf{D}_{e} \nabla u_{e}\right)$.

Here $I_{m}$ can be further expressed as $I_{m}=C_{m} d u / d t+I_{\text {ion }}(u, \boldsymbol{w}), C_{m}$ being a capacitance and $I_{i o n}$ the ionic current, depending on the potential $u$ and on suitable ionic variables that we denote by $\boldsymbol{w}$. The dependence of $I_{i o n}$ on $u$ and $\boldsymbol{w}$ has been described in two different ways in the literature. One approach is based on a precise description of ionic channels (see $[15,24,32]$ ). In this case $\boldsymbol{w}$ represents a vector composed of gate variables and the ions concentration in the cell. The second approach is based on a purely phenomenological evidence (see $[11,23]$ ). In this case $\boldsymbol{w}$ represents a scalar variable called recovery variable. Independently of a specific choice for the ionic model, the complete Bidomain system reads

$\chi C_{m}\left[\begin{array}{cc}1 & -1 \\ -1 & 1\end{array}\right] \frac{\partial}{\partial t}\left[\begin{array}{l}u_{i} \\ u_{e}\end{array}\right]-\left[\begin{array}{c}\nabla \cdot \mathbf{D}_{i} \nabla u_{i} \\ \nabla \cdot \mathbf{D}_{e} \nabla u_{e}\end{array}\right]+\chi\left[\begin{array}{c}I_{\text {ion }}(u, \mathbf{w}) \\ -I_{\text {ion }}(u, \mathbf{w})\end{array}\right]=\left[\begin{array}{c}I_{i}^{\text {app }} \\ -I_{e}^{\text {app }}\end{array}\right]$,

where $I_{\tau}^{\mathrm{app}}(\tau=i, e)$ represent applied external stimuli. The problem is completed by an initial condition $u(\boldsymbol{x}, 0)=u_{0}$ and boundary conditions on $\partial \Omega$. In particular, homogeneous Neumann boundary conditions

$\mathbf{n}^{T} \mathbf{D}_{i} \nabla u_{i}(\boldsymbol{x}, t)=0 \quad$ and $\quad \mathbf{n}^{T} \mathbf{D}_{e} \nabla u_{e}(\boldsymbol{x}, t)=0 \quad$ on $\partial \Omega \times(0, T)$ correspond to an insulated myocardium (here $\mathbf{n}$ is the unit normal outward-pointing vector on the surface). As a consequence of the Gauss theorem, the applied external stimuli must fulfill the compatibility condition

$\int_{\Omega} I_{i}^{\mathrm{app}} d \boldsymbol{x}=\int_{\Omega} I_{e}^{\mathrm{app}} \mathrm{d} \boldsymbol{x}$.

In system (3) the transmembrane potential $u$ is uniquely determined, while the intra- and extracellular potentials $u_{i}$ and $u_{e}$ are determined up to the same function of time. Usually uniqueness is forced by requiring that $u_{e}$ has zero average on $\Omega$. Let us define $\boldsymbol{V}=H^{1}(\Omega) \times H^{1}(\Omega) \backslash\{[c, c]: c \in \mathbb{R}\}$ and denote by $(\cdot, \cdot)$ the scalar product in $L^{2}$. The variational form of the Bidomain problem reads as follows: given $I_{\tau}^{\text {app }}$ and $I_{\text {ion }}$, find $\left[u_{i}, u_{e}\right] \in \boldsymbol{V}$ such that

$$
\begin{aligned}
& \chi C_{m}\left(\frac{\partial u}{\partial t}, \phi\right)+a_{i}\left(u_{i}, \phi_{i}\right)+a_{e}\left(u_{e}, \phi_{e}\right)+\left(I_{\text {ion }}(u), \phi\right) \\
& \quad=\left(I_{i}^{\mathrm{app}}, \phi_{i}\right)-\left(I_{e}^{\mathrm{app}}, \phi_{e}\right)
\end{aligned}
$$

for each $\left[\phi_{i}, \phi_{e}\right] \in \boldsymbol{V}$, where $\phi=\phi_{i}-\phi_{e}$. The forms $a_{\tau}(v, \phi)$ are defined as $a_{\tau}(v, \phi)=\int_{\Omega} \nabla v^{T} D_{\tau} \nabla \phi d \boldsymbol{x}$ (for $\tau=i, e$ ). For well-posedness analysis of the Bidomain problem coupled to the Fitzhugh Nagumo ionic model we refer to [9,29].

The Monodomain problem has been proposed as a simplification of the Bidomain one. It can be deduced in different ways (see $[5,14,16])$. One consists in assuming $\mathbf{D}_{e}=\lambda \mathbf{D}_{i}$, where $\lambda$ is a constant to be properly chosen. Thanks to this assumption, a linear combination of the Bidomain equations with coefficients $\frac{\lambda}{1+\lambda}$ and $-\frac{1}{1+\lambda}$ yields the Monodomain model

$$
\begin{cases}\chi C_{m} \frac{\partial u}{\partial t}-\nabla \cdot\left(\mathbf{D}^{M} \nabla u\right)+\chi I_{\text {ion }}(u)=I^{\text {app }} & \text { in } \Omega \times(0, T), \\ u(\boldsymbol{x}, t=0)=u_{0} & \text { in } \Omega, \\ \mathbf{n}^{T} \mathbf{D}^{M} \nabla u=0 & \text { on } \partial \Omega \times(0, T),\end{cases}
$$

where $\mathbf{D}^{M}=\frac{\lambda \mathbf{D}_{i}}{1+\lambda}$ and $I^{\text {app }}=\frac{\lambda l_{i}^{\text {app }}+I_{e}^{\text {app }}}{1+\lambda}$. The variational form of (7) reads: given $I^{\text {app }}$, and $I_{\text {ion }}$ find $u \in H^{1}(\Omega)$ such that

$\chi C_{m}\left(\frac{\partial u}{\partial t}, \phi\right)+a_{M}(u, \phi)+\left(I_{\text {ion }}(u), \phi\right)=\left(I^{\text {app }}, \phi\right)$

for each $\phi \in H^{1}(\Omega)$. The form $a_{M}(v, \phi):=\int_{\Omega} \nabla v^{T} \mathbf{D}^{M} \nabla \phi d \boldsymbol{x}$ is bilinear, continuous and weakly coercive on $H^{1}(\Omega) \times H^{1}(\Omega)$. For well-posedness analysis of this problem, we still refer to [9]. The Monodomain model is a single parabolic reaction-diffusion PDE for the transmembrane potential, replacing the two equations of the original model. However, this model is not able to capture some physiological and pathological patterns of the action potential propagation (see [6]).

Since our approach is to combine in the same computation both the Bidomain and Monodomain models, we reformulate them in a different way, so that it is easier to downscale the former to the latter, as it was already done in [12] with the purpose of defining a "Monodomain" preconditioner of the Bidomain system. Setting $\widetilde{I}^{\mathrm{app}}=I_{i}^{\mathrm{app}}-I_{e}^{\mathrm{app}}$, by linear combinations of the Eq. (3), we obtain

$$
\left\{\begin{array}{l}
\chi C_{m} \frac{\partial u}{\partial t}-\nabla \cdot\left(\frac{\lambda \mathbf{D}_{i}}{1+\lambda} \nabla u\right)-\nabla \cdot\left(\frac{\lambda \mathbf{D}_{i}-\mathbf{D}_{e}}{1+\lambda} \nabla u_{e}\right)+\chi I_{\text {ion }}(u)=I^{\mathrm{app}}, \\
-\nabla \cdot\left[\mathbf{D}_{i} \nabla u+\left(\mathbf{D}_{i}+\mathbf{D}_{e}\right) \nabla u_{e}\right]=\widetilde{I}^{\text {app }}
\end{array}\right.
$$

More precisely, the first equation in (9) is obtained by linear combination with coefficients $\frac{\lambda}{1+\lambda}$ and $-\frac{1}{1+\lambda}$. The second equation is obtained summing the two equations in (3). The same linear combination leading to (9), together with the assumption $\mathbf{D}_{e}=\lambda \mathbf{D}_{i}$ in the first equation yields the Extended Monodomain formulation in terms of the variables $u$ and $u_{e}$

$$
\left\{\begin{array}{l}
\chi C_{m} \frac{\partial u}{\partial t}-\nabla \cdot\left(\frac{\lambda \mathbf{D}_{i}}{1+\lambda} \nabla u\right)+\chi I_{\text {ion }}(u)=I^{\mathrm{app}} \\
-\nabla \cdot\left[\mathbf{D}_{i} \nabla u+\left(\mathbf{D}_{i}+\mathbf{D}_{e}\right) \nabla u_{e}\right]=\widetilde{I}^{\mathrm{app}}
\end{array}\right.
$$


As for the Bidomain model, also in the Extended Monodomain model (10) the intra- and extracellular potentials $u_{i}$ and $u_{e}$ are defined only up to the same function of time. Again, we will fix such function by requiring that $u_{e}$ has zero average.

Notice that our formulation of the Monodomain model comes immediately from (9) when the differential term in $u_{e}$ in the first equation is dropped.

Our proposed Hybridomain models consists in splitting the domain $\Omega$ into two parts $\Omega_{B}$ and $\Omega_{M}$, such that $\Omega_{M} \cup \Omega_{B}=\Omega$ and $\Omega_{M} \cap \Omega_{B}=\varnothing$ and setting the problem

$$
\left\{\begin{array}{l}
\chi C_{m} \frac{\partial u}{\partial t}-\nabla \cdot\left(\frac{\lambda \mathbf{D}_{i}}{1+\lambda} \nabla u\right)-\nabla \cdot\left(\frac{\left(\lambda \mathbf{D}_{i}-\mathbf{D}_{e}\right) \Omega_{\Omega_{B}}}{1+\lambda} \nabla u_{e}\right)+\chi I_{\text {ion }}(u)=I^{\mathrm{app}} \\
-\nabla \cdot\left[\mathbf{D}_{i} \nabla u+\left(\mathbf{D}_{i}+\mathbf{D}_{e}\right) \nabla u_{e}\right]=\widetilde{I}^{\mathrm{app}}
\end{array}\right.
$$

where $1_{\Omega_{B}}$ is the characteristic function defined in $\Omega$, so that $1_{\Omega_{B}}(x, y, z)=1$ for $x, y, z \in \Omega_{B}$ and $1_{\Omega_{B}}(x, y, z)=0$ elsewhere. At each time step, on the basis of our error estimator, we adapt $\Omega_{B}$ as described in Section 4.

Remark. In the sequel we introduce the following notation:

$\left\|u_{i}, u_{e}\right\|_{E(\Omega)}^{2} \equiv \int_{\Omega}\left(\nabla u_{i}\right)^{T} \mathbf{D}_{i} \nabla u_{i}+\int_{\Omega}\left(\nabla u_{e}\right)^{T} \mathbf{D}_{e} \nabla u_{e}$

which is a semi-norm in $V$ since the two tensors $\mathbf{D}_{i}$ and $\mathbf{D}_{e}$ are assumed to be positive. Starting from (6), assuming the intra- and extracellular applied currents to be equal and taking $\phi_{i}=u_{i}$ and $\phi_{e}=u_{e}$, so that $\phi=u$, we obtain

$\frac{\chi C_{m}}{2} \int_{\Omega} \frac{\partial u^{2}}{\partial t}+\left\|u_{i}, u_{e}\right\|_{E(\Omega)}^{2}=-\left(I_{\text {ion }}(u), u\right)+\left(I_{i}^{\mathrm{app}}, u\right)$.

This equation states the balance of energy of the considered physical system, the first term on the right-hand side being the energy rate due to ionic currents and the second term on the right-hand side being the energy rate due to external currents. Given a specific form of the ionic model, an estimate of the energy rate by ionic currents in terms of the solution $u$ can be deduced.

\subsection{Semi-discretization of the problem}

We consider a semi-implicit first order time advancing scheme, where the terms depending on the ionic currents are taken at the previous time step, so that at each time step the problem is linear. Stability bounds induced by this choice are in general not too restrictive in practice. Let $\Delta t$ be the (constant) time step of the discretization. Denote with superscript $n$ the variables computed at time $t^{n}=t^{0}+n \Delta t$. We denote by $\left(u_{i, B}^{n}, u_{e, B}^{n}, u_{B}^{n}=u_{i, B}^{n}-u_{e, B}^{n}\right)$ the solution to (9), with $\left(u_{i, M}^{n}, u_{e, M}^{n}, u_{M}^{n}=u_{i, M}^{n}-u_{e, M}^{n}\right)$ the solution to (10) and with $\left(u_{i, H}^{n}, u_{e, H}^{n}, u_{H}^{n}=u_{i, H}^{n}-u_{e, H}^{n}\right)$ the solution to the Hybridomain problem (11). Moving from time step $t^{n}$ to $t^{n+1}$ the semiimplicit time-discretization of (9) reads

$$
\left\{\begin{array}{l}
\chi C_{m} \frac{u_{B}^{n+1}-u_{B}^{n}}{\Delta t}-\nabla \cdot\left(\frac{\lambda \mathbf{D}_{i}}{1+\lambda} \nabla u_{B}^{n+1}\right)-\nabla \cdot\left(\frac{\lambda \mathbf{D}_{i}-\mathbf{D}_{e}}{1+\lambda} \nabla u_{e, B}^{n+1}\right) \\
\quad+\chi I_{\text {ion }}\left(u_{B}^{n}\right)=I^{\mathrm{app}, n+1} \\
-\nabla \cdot\left[\mathbf{D}_{i} \nabla u_{B}^{n+1}+\left(\mathbf{D}_{i}+\mathbf{D}_{e}\right) \nabla u_{e, B}^{n+1}\right]=\widetilde{I}^{\mathrm{app}, n+1} .
\end{array}\right.
$$

Similarly, for the Hybridomain (11), we resort to the following discretization:

$$
\left\{\begin{array}{l}
\chi C_{m} \frac{u_{H}^{n+1}-u_{H}^{n}}{\Delta t}-\nabla \cdot\left(\frac{\lambda \mathbf{D}_{i}}{1+\lambda} \nabla u_{H}^{n+1}\right) \\
-\nabla \cdot\left(\frac{\left(\lambda \mathbf{D}_{i}-\mathbf{D}_{e}\right) \Omega_{\Omega_{B}^{n}}^{n}}{1+\lambda} \nabla u_{e, H}^{n+1}\right)=I^{\mathrm{app}, n+1}-\chi I_{\mathrm{ion}}\left(u_{H}^{n}\right), \\
-\nabla \cdot\left[\mathbf{D}_{i} \nabla u_{H}^{n+1}+\left(\mathbf{D}_{i}+\mathbf{D}_{e}\right) \nabla u_{e, H}^{n+1}\right]=\widetilde{I}^{\mathrm{app}, n+1} .
\end{array}\right.
$$

Notice the choice $1_{\Omega_{B}^{n}}$, which implies that the region where we switch the "Bidomain" term on is estimated upon the solution at the previous time step. A similar discretization is carried out for the Monodomain model (10).

After discretizing (14) in space for instance by Lagrange finite elements, we are led to an algebraic system of the form

$$
\left[\begin{array}{ll}
\mathbf{B}_{u u} & \mathbf{B}_{u e} \\
\mathbf{B}_{e u} & \mathbf{B}_{e e}
\end{array}\right]\left[\begin{array}{l}
\mathbf{u}_{B}^{n+1} \\
\mathbf{u}_{B, e}^{n+1}
\end{array}\right]=\left[\begin{array}{l}
\mathbf{f}^{n+1} \\
\mathbf{g}^{n+1}
\end{array}\right]
$$

$\mathbf{u}_{B}^{n+1}$ and $\mathbf{u}_{e, B}^{n+1}$ being the vectors of nodal values corresponding to $u_{B}^{n+1}$ and $u_{e, B}^{n+1}$, respectively. Similarly, the Monodomain problem will read at algebraic level

$$
\left[\begin{array}{ll}
\mathbf{B}_{u u} & \\
\mathbf{B}_{e u} & \mathbf{B}_{e e}
\end{array}\right]\left[\begin{array}{l}
\mathbf{u}_{M}^{n+1} \\
\mathbf{u}_{M, e}^{n+1}
\end{array}\right]=\left[\begin{array}{l}
\mathbf{f}^{n+1} \\
\mathbf{g}^{n+1}
\end{array}\right]
$$

In the Hybridomain approach we will assemble the off diagonal matrix $\mathbf{B}_{u e}$ only in those elements $K \in \Omega_{B}$.

\section{The a posteriori estimator}

The differences between the Bidomain and Monodomain solutions at the generic time $t^{n+1}$ are denoted by

$e_{i, M}^{t o t, n+1}=u_{i, B}^{n+1}-u_{i, M}^{n+1}, \quad e_{e, M}^{t o t, n+1}=u_{e, B}^{n+1}-u_{e, M}^{n+1}$,
$e_{u, M}^{t o t, n+1}=u_{B}^{n+1}-u_{M}^{n+1}$,

while

$$
\begin{aligned}
& e_{i, H}^{t o t, n+1}=u_{i, B}^{n+1}-u_{i, H}^{n+1}, \quad e_{e, H}^{t o t, n+1}=u_{e, B}^{n+1}-u_{e, H}^{n+1}, \\
& e_{u, H}^{t o t, n+1}=u_{B}^{n+1}-u_{H}^{n+1}
\end{aligned}
$$

denote the differences between the Bidomain and the Hybridomain models. In the sequel we set $\mathbf{D}_{\varepsilon} \equiv \frac{\mathbf{D}_{e}-\lambda \mathbf{D}_{i}}{1+\lambda}$.

We split the differences (17) (resp. (16)) into two components. Let $\left(\tilde{u}_{B}^{n+1}, \tilde{u}_{e, B}^{n+1}, \tilde{u}_{i, B}^{n+1}\right)$ be the solution of (14) from time step $t^{n}$ to $t^{n+1}$ moving from the Hybridomain (resp. Monodomain) solution at time $t^{n}$, namely

$$
\left\{\begin{array}{l}
\chi C_{m} \frac{\tilde{u}_{B}^{n+1}-\tilde{u}_{B}^{n}}{\Delta t}-\nabla \cdot\left(\frac{\lambda \mathbf{D}_{i}}{1+\lambda} \nabla \tilde{u}_{B}^{n+1}\right)-\nabla \cdot\left(\frac{\lambda \mathbf{D}_{i}-\mathbf{D}_{e}}{1+\lambda} \nabla \tilde{u}_{e, B}^{n+1}\right) \\
\quad+\chi I_{\text {ion }}\left(\tilde{u}_{B}^{n}\right)=I^{\mathrm{app}, n+1} \\
-\nabla \cdot\left[\mathbf{D}_{i} \nabla \tilde{u}_{B}^{n+1}+\left(\mathbf{D}_{i}+\mathbf{D}_{e}\right) \nabla \tilde{u}_{e, B}^{n+1}\right]=\widetilde{I}^{\mathrm{app}, n+1} \\
\tilde{u}_{i, B}^{n}=u_{i, H}^{n}, \quad \tilde{u}_{e, B}^{n}=u_{e, H}^{n} .
\end{array}\right.
$$

Then the total error is split as

$$
e_{i, H}^{n+1, t o t}=\tilde{e}_{i, H}^{n+1}+e_{i, H}^{n}, \quad \tilde{e}_{i, H}^{n+1}=u_{i, B}^{n+1}-\tilde{u}_{i, B}^{n+1}, \quad e_{i, H}^{n+1}=\tilde{u}_{i, B}^{n+1}-u_{i, H}^{n+1}
$$

and similarly for the other components $e_{u, H}^{n+1, \text { tot }}$ and $e_{e, H}^{n+1, \text { tot }}$. Component $\left(e_{u, H}^{n+1}, e_{i, H}^{n+1}, e_{e, H}^{n+1}\right)$ can be considered the local contribution to the error, being the difference introduced at each time step by using the Hybridomain model instead of the Bidomain one, starting from the same solution at time $t^{n}$. Contribution $\left(\tilde{e}_{u, H}^{n+1}, \tilde{e}_{i, H}^{n+1}, \tilde{e}_{e, H}^{n+1}\right)$ is a propagated error whose analysis involves the stability of the time discrete Bidomain operator, coupled with the ionic model (see e.g. [2]). Hereafter, we focus on estimating the local error only. Numerical results will show that this achieves an effective control also on the total error.

\subsection{Definition of the estimator}

Recalling that $\Omega \backslash \Omega_{B}=\Omega_{M}$, memberwise substraction of Eqs. (15) to the corresponding ones of (18) yields the error equation 
$\left\{\begin{array}{l}\frac{\chi C_{m}}{\Delta t} e_{u, H}^{n+1}-\nabla \cdot\left(\frac{\lambda \mathbf{D}_{i}}{1+\lambda} \nabla e_{u, H}^{n+1}\right)+\nabla \cdot\left(\mathbf{D}_{\varepsilon} \nabla e_{e, H}^{n+1}\right) \\ \quad=-\nabla \cdot\left(\mathbf{D}_{\varepsilon} 1_{\Omega_{M}^{n}} \nabla u_{e, H}^{n+1}\right), \\ -\nabla \cdot\left[\mathbf{D}_{i} \nabla e_{u, H}^{n+1}+\left(\mathbf{D}_{i}+\mathbf{D}_{e}\right) \nabla e_{e, H}^{n+1}\right]=0,\end{array}\right.$

which can be written in equivalent form, upon taking a linear combination of the two equations with coefficients $(1,1 /(1+\lambda))$ as

$$
\left\{\begin{array}{l}
\frac{\chi C_{m}}{\Delta t} e_{u, H}^{n+1}-\nabla \cdot\left(\mathbf{D}_{i} \nabla\left(e_{u, H}^{n+1}+e_{e, H}^{n+1}\right)\right) \\
\quad=-\nabla \cdot\left(\mathbf{D}_{\varepsilon} 1_{\Omega_{M}^{n}} \nabla u_{e, H}^{n+1}\right), \\
-\nabla \cdot\left[\mathbf{D}_{i} \nabla e_{u, H}^{n+1}+\left(\mathbf{D}_{i}+\mathbf{D}_{e}\right) \nabla e_{e, H}^{n+1}\right]=0 .
\end{array}\right.
$$

In the sequel we drop time index $n+1$ for the sake of notation. Notice that if in the previous system we force $\Omega_{M}^{n}=\Omega$ we estimate the errors $e_{u, M}, e_{i, M}, e_{e, M}$.

Let us multiply the first equation in the previous system by $e_{u, H}$ and the second by $e_{e, H}$, integrate over $\Omega$ and sum the two equations. We obtain

$$
\begin{aligned}
\int_{\Omega} & \frac{\chi C_{m}}{\Delta t} e_{u, H}^{2}+\int_{\Omega} \nabla e_{u, H}^{T} \mathbf{D}_{i} \nabla e_{i, H}-\int_{\partial \Omega} \mathbf{n}^{T} \mathbf{D}_{i} \nabla e_{i, H} e_{u, H} \\
& +\int_{\Omega} \nabla e_{e, H}^{T} \mathbf{D}_{i} \nabla e_{u, H}-\int_{\partial \Omega} \mathbf{n}^{T} \mathbf{D}_{i} \nabla e_{u, H} e_{e, H}+\int_{\Omega} \nabla e_{e, H}^{T}\left(\mathbf{D}_{i}+\mathbf{D}_{e}\right) \nabla e_{e, H} \\
& -\int_{\partial \Omega} \mathbf{n}^{T}\left(\mathbf{D}_{i}+\mathbf{D}_{e}\right) \nabla e_{e, H} e_{e, H} \\
& =\int_{\Omega_{M}^{n}} \nabla e_{u, H}^{T} \mathbf{D}_{\varepsilon} \nabla u_{e, H}-\int_{\partial \Omega_{M}^{n} \cap \partial \Omega} \mathbf{n}^{T} \mathbf{D}_{\varepsilon} \nabla u_{e, H} e_{u, H}
\end{aligned}
$$

We assume that both problems fulfill the same conditions on the boundary $\partial \Omega$. For this reason, in the previous equation we drop the integrals on $\partial \Omega$.

Exploiting $e_{u, H}=e_{i, H}-e_{e, H}$, we have

$$
\begin{gathered}
\int_{\Omega} \nabla e_{u, H}^{T} \mathbf{D}_{i} \nabla e_{i, H}+\int_{\Omega} \nabla e_{e, H}^{T} \mathbf{D}_{i} \nabla e_{u, H}+\int_{\Omega} \nabla e_{e, H}^{T}\left(\mathbf{D}_{i}+\mathbf{D}_{e}\right) \nabla e_{e, H} \\
=\int_{\Omega} \nabla e_{i, H}^{T} \mathbf{D}_{i} \nabla e_{i, H}+\int_{\Omega} \nabla e_{e, H}^{T} \mathbf{D}_{e} \nabla e_{e, H},
\end{gathered}
$$

so that the variational formulation of the error system reads

$$
\begin{gathered}
\int_{\Omega} \frac{\chi C_{m}}{\Delta t} e_{u, H}^{2}+\int_{\Omega} \nabla e_{i, H}^{T} \mathbf{D}_{i} \nabla e_{i, H}+\int_{\Omega} \nabla e_{e, H}^{T} \mathbf{D}_{e} \nabla e_{e, H} \\
=\int_{\Omega_{M}^{n}} \nabla e_{i, H}^{T} \mathbf{D}_{\varepsilon} \nabla u_{e, H}-\int_{\Omega_{M}^{n}} \nabla e_{e, H}^{T} \mathbf{D}_{\varepsilon} \nabla u_{e, H} .
\end{gathered}
$$

Using standard techniques, since $\mathbf{D}_{i}$ and $\mathbf{D}_{e}$ are symmetric and positive definite, we can manipulate the right-hand side of the previous equation as follows:

$$
\begin{aligned}
\int_{\Omega_{M}^{n}} & \nabla e_{i, H}^{T} \mathbf{D}_{\varepsilon} \nabla u_{e, H}-\int_{\Omega_{M}^{n}} \nabla e_{e, H}^{T} \mathbf{D}_{\varepsilon} \nabla u_{e, H} \\
\leqslant & \int_{\Omega_{M}^{n}}\left|\nabla e_{i, H}^{T} \mathbf{D}_{i}^{1 / 2} \mathbf{D}_{i}^{-1 / 2} \mathbf{D}_{\varepsilon} \nabla u_{e, H}\right| \\
& \quad+\int_{\Omega_{M}^{n}}\left|\nabla e_{e, H}^{T} \mathbf{D}_{e}^{1 / 2} \mathbf{D}_{e}^{-1 / 2} \mathbf{D}_{\varepsilon} \nabla u_{e, H}\right| \\
\leqslant & \frac{1}{2}\left\|e_{i, H}, e_{e, H}\right\|_{E\left(\Omega_{M}^{n}\right)}^{2}+\frac{1}{2} \int_{\Omega_{M}^{n}} \nabla u_{e, H}^{T} \mathbf{D}_{\varepsilon}^{T}\left(\mathbf{D}_{i}^{-1}+\mathbf{D}_{e}^{-1}\right) \mathbf{D}_{\varepsilon} \nabla u_{e, H}
\end{aligned}
$$

yielding

$$
\begin{aligned}
& \int_{\Omega} \frac{\chi C_{m}}{\Delta t} e_{u, H}^{2}+\frac{1}{2}\left\|e_{i, H}, e_{e, H}\right\|_{E\left(\Omega_{M}^{n}\right)}^{2}+\left\|e_{i, H}, e_{e, H}\right\|_{E\left(\Omega_{B}^{n}\right)}^{2} \\
& \quad \leqslant \frac{1}{2} \int_{\Omega_{M}} \nabla u_{e, H}^{T} \mathbf{D}_{\varepsilon}^{T}\left(\mathbf{D}_{i}^{-1}+\mathbf{D}_{e}^{-1}\right) \mathbf{D}_{\varepsilon} \nabla u_{e, H} \equiv \eta_{\Omega_{M}}^{2}\left(u_{e, H}\right) .
\end{aligned}
$$

In the sequel we denote

$$
\left.\left\|e_{i, H}, e_{e, H}\right\|\right|^{2} \equiv \int_{\Omega} \frac{\chi C_{m}}{\Delta t} e_{u, H}^{2}+\frac{1}{2}\left\|e_{i, H}, e_{e, H}\right\|_{E\left(\Omega_{M}^{n}\right)}^{2}+\left\|e_{i, H}, e_{e, H}\right\|_{E\left(\Omega_{B}^{n}\right)}^{2},
$$

which is still a norm, so we can write in short

$\left\|e_{i, H}, e_{e, H}\right\| \leqslant \eta_{\Omega_{M}}\left(u_{e, H}\right)$.

The quantity $\eta_{\Omega_{M}}\left(u_{e, H}\right)$ bounds therefore the "local" difference between the "template" Bidomain model and the Hybridomain solution currently computed.

Remark. Notice that with similar arguments it is possible to prove that the "complementary" estimator

$\eta_{\Omega_{B}}^{2}\left(u_{e, H}\right)=\frac{1}{2} \int_{\Omega_{B}} \nabla u_{e, H}^{T} \mathbf{D}_{\varepsilon}^{T}\left(\mathbf{D}_{i}^{-1}+\mathbf{D}_{e}^{-1}\right) \mathbf{D}_{\varepsilon} \nabla u_{e, H}$

measures the difference between the Hybridomain solution and the Monodomain one.

Remark. The total error could be obviously split in different ways. In particular, we could split the total error into a local component obtained by solving a time step of Hybridomain system moving from the "exact" Bidomain solution at the previous time step. This is the classical approach in analyzing Ordinary Differential Equations schemes. Following this splitting, we can perform an analysis similar to the one carried out above, yielding an upper bound for the local error given by $\eta_{\Omega_{\mathrm{M}}}\left(\bar{u}_{e, H}\right)$ where $\bar{u}_{e, H}$ is the solution of the Hybridomain system computed starting from the Bidomain data at the previous time step. Differently than $u_{e, H}$ in (24), $\bar{u}_{e, H}$ is not available in current applications (since one needs to know the Bidomain solution that we do not want to compute actually). For this reason we prefer to consider our error splitting leading to (24).

\subsection{An error lower bound}

Let us start from the error equation (22). Observe that the left hand side can be derived from a bilinear symmetric scalar form, that we denote by

$$
\begin{aligned}
\left\langle\left[e_{i, H}, e_{e, H}\right],\left[v_{i, H}, v_{e, H}\right]\right\rangle_{\dagger} \equiv & \int_{\Omega} \frac{\chi C_{m}}{\Delta t}\left(e_{i, H}-e_{e, H}\right)\left(v_{i, H}-v_{e, H}\right) \\
& +\int_{\Omega} \nabla v_{i, H}^{T} \mathbf{D}_{i} \nabla e_{i, H}+\int_{\Omega} \nabla v_{e, H}^{T} \mathbf{D}_{e} \nabla e_{e, H} .
\end{aligned}
$$

The associated norm is denoted by

$$
\left.\left\|e_{i, H}, e_{e, H}\right\|\right|_{\dagger} ^{2} \equiv \int_{\Omega} \frac{\chi C_{m}}{\Delta t} e_{u, H}^{2}+\int_{\Omega} \nabla e_{i, H}^{T} \mathbf{D}_{i} \nabla e_{i, H}+\int_{\Omega} \nabla e_{e, H}^{T} \mathbf{D}_{e} \nabla e_{e, H} .
$$

Notice the equivalence between the two norms $\||| \cdot|| \mid$ and $\||\cdot|\|_{\dagger}$. It is actually verified by direct inspection that

$\left.\frac{1}{2}\left\|e_{i, H}, e_{e, H}\right\|\right|_{\dagger} ^{2} \leqslant\left\|\left|e_{i, H}, e_{e, H}\left\|\left.\right|^{2} \leqslant\right\| e_{i, H}, e_{e, H} \|\right|_{\dagger}^{2}\right.$.

By exploiting the properties of the scalar product, and denoting

$\mathcal{F}\left(\left[v_{i, H}, v_{e, H}\right]\right) \equiv \int_{\Omega_{M}^{n}} \nabla v_{i, H}^{T} \mathbf{D}_{\varepsilon} \nabla u_{e, H}-\int_{\Omega_{M}^{n}} \nabla v_{e, H}^{T} \mathbf{D}_{\varepsilon} \nabla u_{e, H}$,

we have from (22) that

$$
\begin{aligned}
\left\|e_{i, H}, e_{e, H}\right\|_{\dagger}^{2} & =\int_{\Omega_{M}^{n}} \nabla e_{i, H}^{T} \mathbf{D}_{\varepsilon} \nabla u_{e, H}-\int_{\Omega_{M}^{n}} \nabla e_{e, H}^{T} \mathbf{D}_{\varepsilon} \nabla u_{e, H}=\|\mathcal{F}\|_{\dagger}^{\prime, 2} \\
& =\left(\sup _{\left[v_{i}, v_{e}\right] \neq[0,0]} \frac{\mathcal{F}\left(\left[v_{i, H}, v_{e, H}\right]\right)}{\left\|\mid v_{i, H}, v_{e, H}\right\|_{\dagger}}\right)^{2} .
\end{aligned}
$$


Consequently, for any choice of the test functions $\left[v_{i, H}, v_{e, H}\right]$, we have

$\zeta\left(\left[v_{i, H}, v_{e, H}\right]\right)=\frac{1}{\sqrt{2}} \frac{\left|\mathcal{F}\left(\left[v_{i, H}, v_{e, H}\right]\right)\right|}{||\left|v_{i, H}, v_{e, H}\right| \|_{\dagger}} \leqslant \frac{1}{\sqrt{2}}\left\|\left|e_{i, H}, e_{e, H}\right|\right\|_{\dagger} \leqslant\left\||| e_{i, H}, e_{e, H}\right\| \mid$.

Let us consider the family of test functions of the form $\left[p u_{e, H},-u_{e, H}\right]$. In order to have a sharp lower bound, we look for the parameter $p_{\text {opt }}$ which maximizes $\zeta$.

Upon differentiating $\zeta\left(\left[p u_{e, H},-u_{e, H}\right]\right)^{2}$ with respect to $p$ and equating it to zero, we obtain that the maximum lower bound corresponds to

$p=\frac{\int_{\Omega} \nabla u_{e, H}^{T} \mathbf{D}_{e} \nabla u_{e, H}}{\int_{\Omega} \nabla u_{e, H}^{T} \mathbf{D}_{i} \nabla u_{e, H}} \equiv p_{\mathrm{opt}}$.

Notice that $p_{\text {opt }}$ would be equal to $\lambda$ under Monodomain assumption $\mathbf{D}_{e}=\lambda \mathbf{D}_{i}$.

In the sequel, we set $\zeta_{\text {opt }} \equiv \zeta\left(\left[p_{\text {opt }} u_{e, H},-u_{e, H}\right]\right)$ for the lower bound on the error $\left\|\left|e_{i, H}, e_{e, H} \|\right|\right.$.

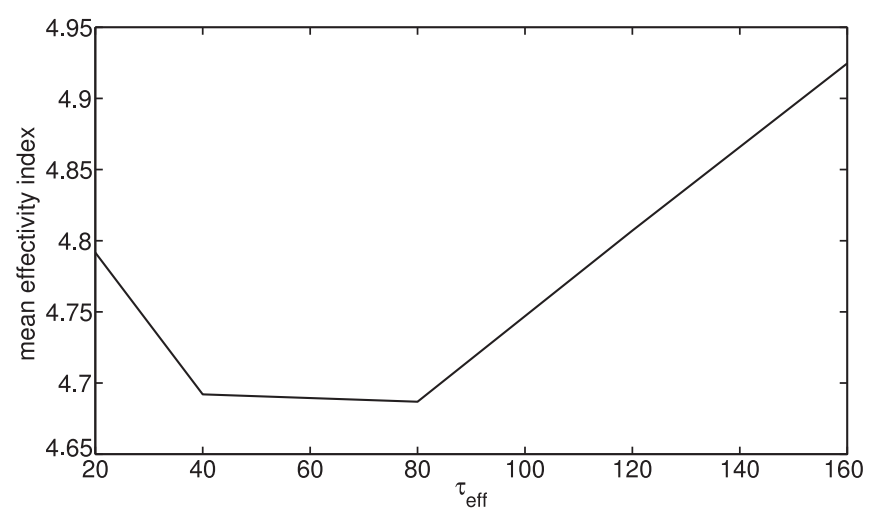

Fig. 2. Average effectivity index for different values of the threshold $\tau_{\text {eff. }}$ The minimum average is reached for $\tau_{\text {eff }}=80$.

\section{The adaptive algorithm}

As we have pointed out previously, in our Hybridomain model the region $\Omega_{B}^{n}$ where the Bidomain model is active is evaluated
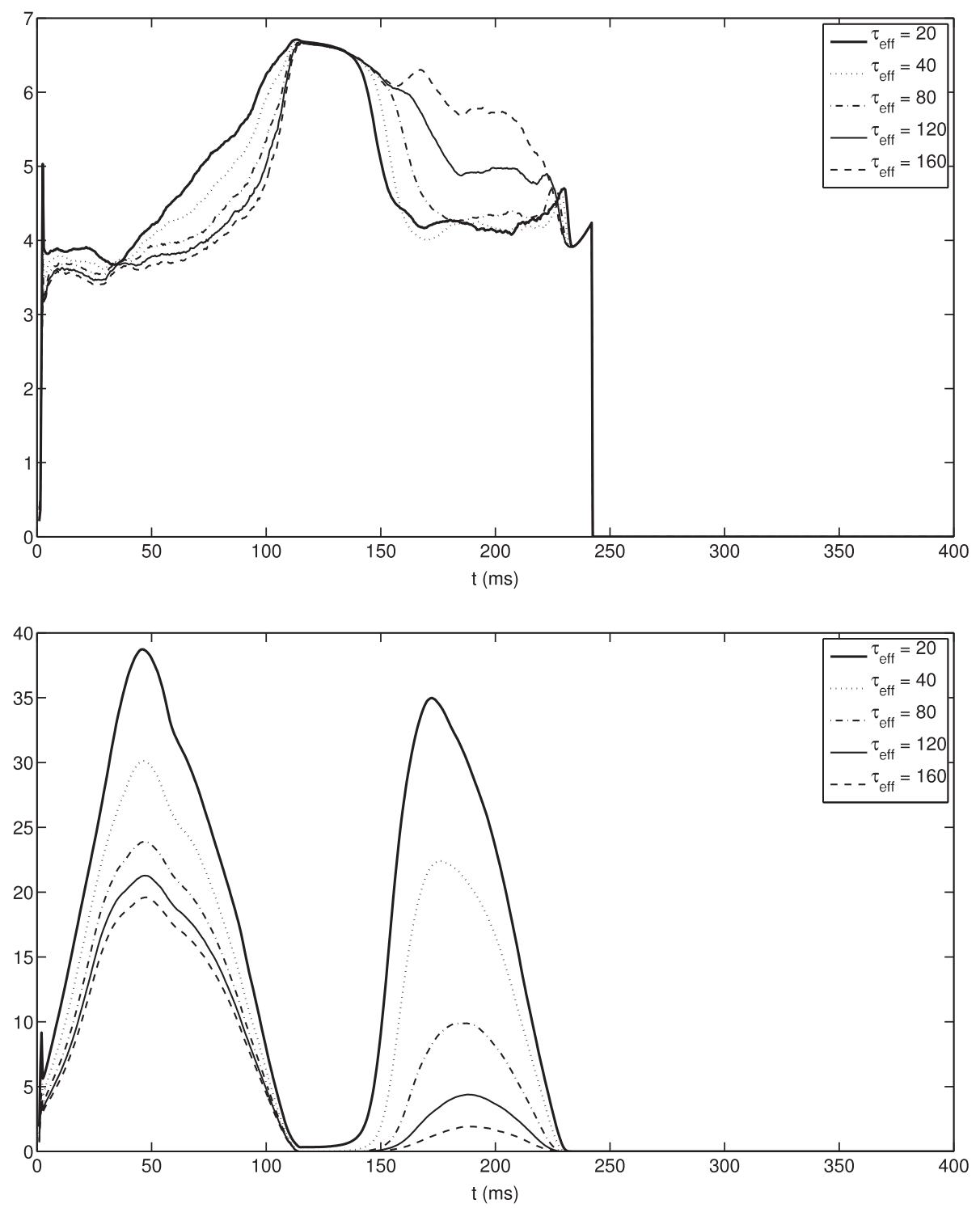

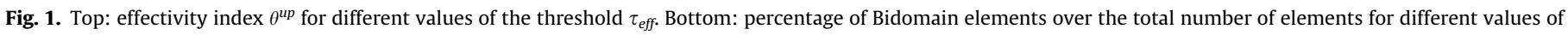
the threshold $\tau_{\text {eff. }}$. 


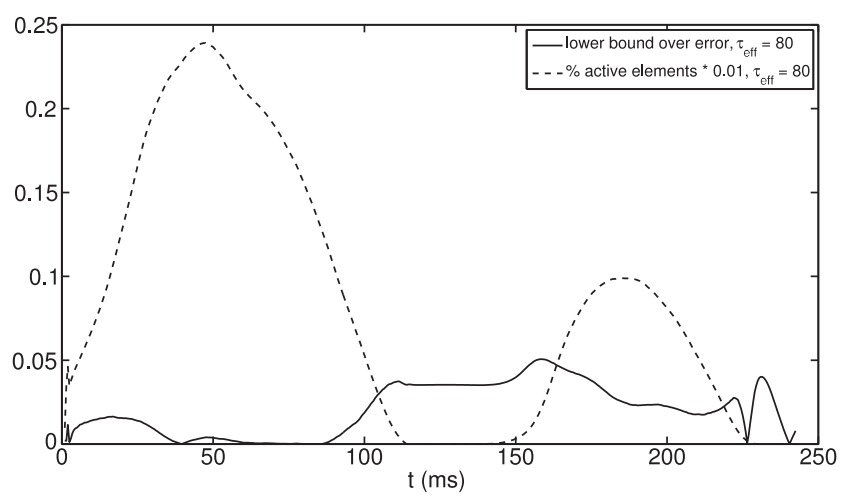

Fig. 3. Effectivity index $\theta^{\text {low }}$ and percentage of active Bidomain elements for $\tau_{\text {eff }}=80$.

on the basis of the estimator. More precisely, we introduce the local error indicator

$\eta_{K}^{2}\left(u_{e, H}\right)=\frac{1}{2} \int_{K} \nabla u_{e, H}^{T} \mathbf{D}_{\varepsilon}^{T}\left(\mathbf{D}_{i}^{-1}+\mathbf{D}_{e}^{-1}\right) \mathbf{D}_{\varepsilon} \nabla u_{e, H}, \quad K \in \mathcal{T}_{h}$

so that, if $N_{M}$ is the number of elements in $\Omega_{M}$ (we drop the time index for the sake of notation)

$\eta_{\Omega_{M}}\left(u_{e, H}\right)=\sqrt{\sum_{K=1}^{N_{M}} \eta_{K}^{2}\left(u_{e, H}\right)}$.
We impose then a uniform distribution of the error among all elements of the mesh $\mathcal{T}_{h}$, namely

$\eta_{K}\left(u_{e, H}\right) \leqslant \frac{\tau}{\sqrt{N}}$

$N$ being the total number of elements and $\tau$ a prescribed tolerance. Observe that this choice guarantees that $\eta_{\Omega_{M}}\left(u_{e, H}\right) \leqslant \tau$. More precisely, the refinement algorithm reads:

case 1 if for $K \in \Omega_{M}^{n}$ inequality (28) is fulfilled, then $K \in \Omega_{M}^{n+1}$, else

case 2 if (28) is not fulfilled, $K \in \Omega_{B}^{n+1}$.

The coarsening strategy is based on the complementary estimator $\eta_{\Omega_{\mathrm{B}}}\left(u_{e, H}\right)$. For $K \in \Omega_{B}$ we compute $\eta_{K}\left(u_{e, H}\right)$. Then, for a given fraction $\sigma$ we verify the inequality

$\eta_{K}\left(u_{e, H}\right) \geqslant \frac{\sigma \tau}{\sqrt{N}}$.

In our numerical tests we used $\sigma=1$. The coarsening strategy reads

case 1 if for $K \in \Omega_{B}^{n}$, inequality (29) is fulfilled, then $K \in \Omega_{B}^{n+1}$, else case 2 if (29) is not fulfilled, $K \in \Omega_{M}^{n+1}$.

It is worth pointing out that in this adaptive strategy the upper right block of the matrix needs to be reassembled at each time step. As we will see in Section 5, the adaptive strategy is still faster than the full Bidomain solver.

The adaptive algorithm has been implemented within the finite elements library LifeV (see www.lifev.org). The space discretization chosen is a piecewise linear finite element discretization while the time advancing scheme is described in Section 2.1.
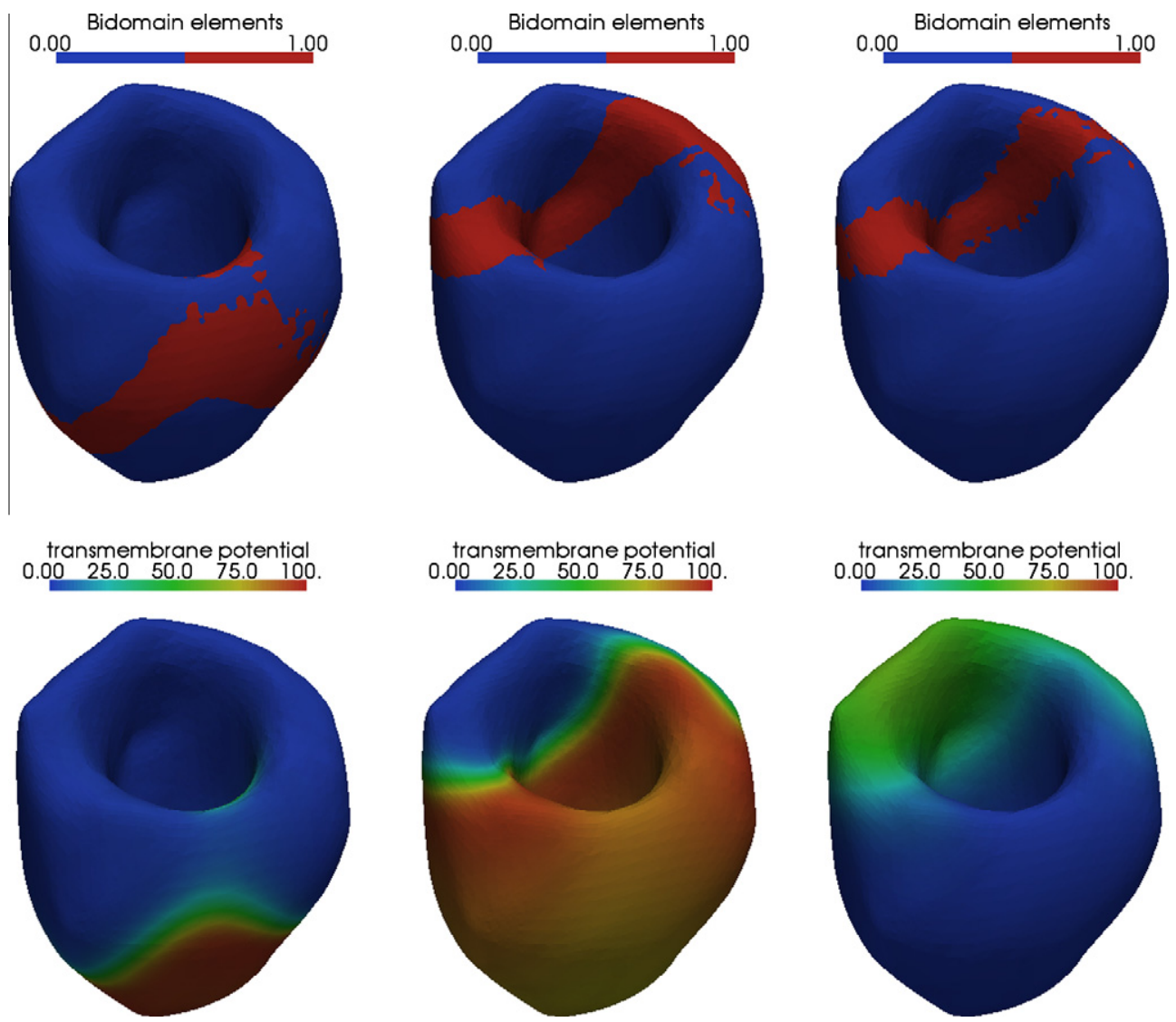

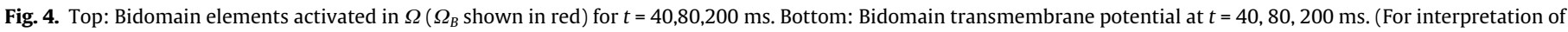
the references to colour in this figure legend, the reader is referred to the web version of this article.) 
The solution at each time step of the Hybridomain model is carried out with the Trilinos linear solver GMRES or Flexible GMRES [25], implemented in Belos package (http://trilinos.sandia.gov). The system is preconditioned with the Extended Monodomain mod$\mathrm{el}$, as done in [12], with different values of the inner tolerance. The same strategy has been applied to the Bidomain system, to compare performances and results. The Extended Monodomain system is solved blockwise, using ILU-preconditioned CG solver for each block.

Remark. The estimator $\eta_{\Omega_{M}}\left(u_{e, H}\right)$ and the lower bound $\zeta_{\text {opt }}\left(u_{e, H}\right)$ provide bounds for the local error. Consistently, in the next section we investigate efficiency and reliability of the estimator by comparing the Hybridomain solution at time $t^{n+1}$ with the Bidomain solution at the same time step, initialized to the same datum at time $t^{n}$. In the numerical results presented in Section 5 we have decided to initialize both the Bidomain and Hybridomain solvers to the Bidomain solution at each time step.

\section{Numerical results}

We first address the comparison of Bidomain, Monodomain and Hybridomain solvers for the propagation of the action potential in a region of the left ventricle. This geometry has been segmented

\section{Table 1}

Percentage gain in CPU effort using the adaptive strategy with respect to solving the Bidomain system. We report in the first row the average gain in iteration count; in the second row the average gain in CPU time required for the solution of the linear system; in the third row the average net gain in CPU time, considering the assembling time required by the Hybridomain.

\begin{tabular}{ll}
\hline$g_{\text {it }}$ & $28.6 \%$ \\
$g_{\text {time }}$ & $35.0 \%$ \\
$g_{\text {time, net }}$ & $33.4 \%$ \\
\hline
\end{tabular}

from SPECT images provided by Dr. E.V. Garcia (Emory Hospital, Atlanta, GA, USA) [1,4] using the Level Set method implemented in the VMTK code (see www.vmtk.org). The computational mesh features 1233256 elements and 199766 degrees of freedom. The conductivity of the tissue in this test case is homogeneous non-isotropic due to the presence of the cardiac fibers, as described in Section 2. Details on the conductivity parameters and the analytical description of the fibers used for the numerical experiments in Section 5.1 can be found in [7]. More precisely, the geometrical parameters of the fiber description have been here adjusted to fit the size of the geometry at hand.

In Section 5.2 we simulate the presence of a scar in the ventricle wall and we compare the pattern obtained with the different solv-
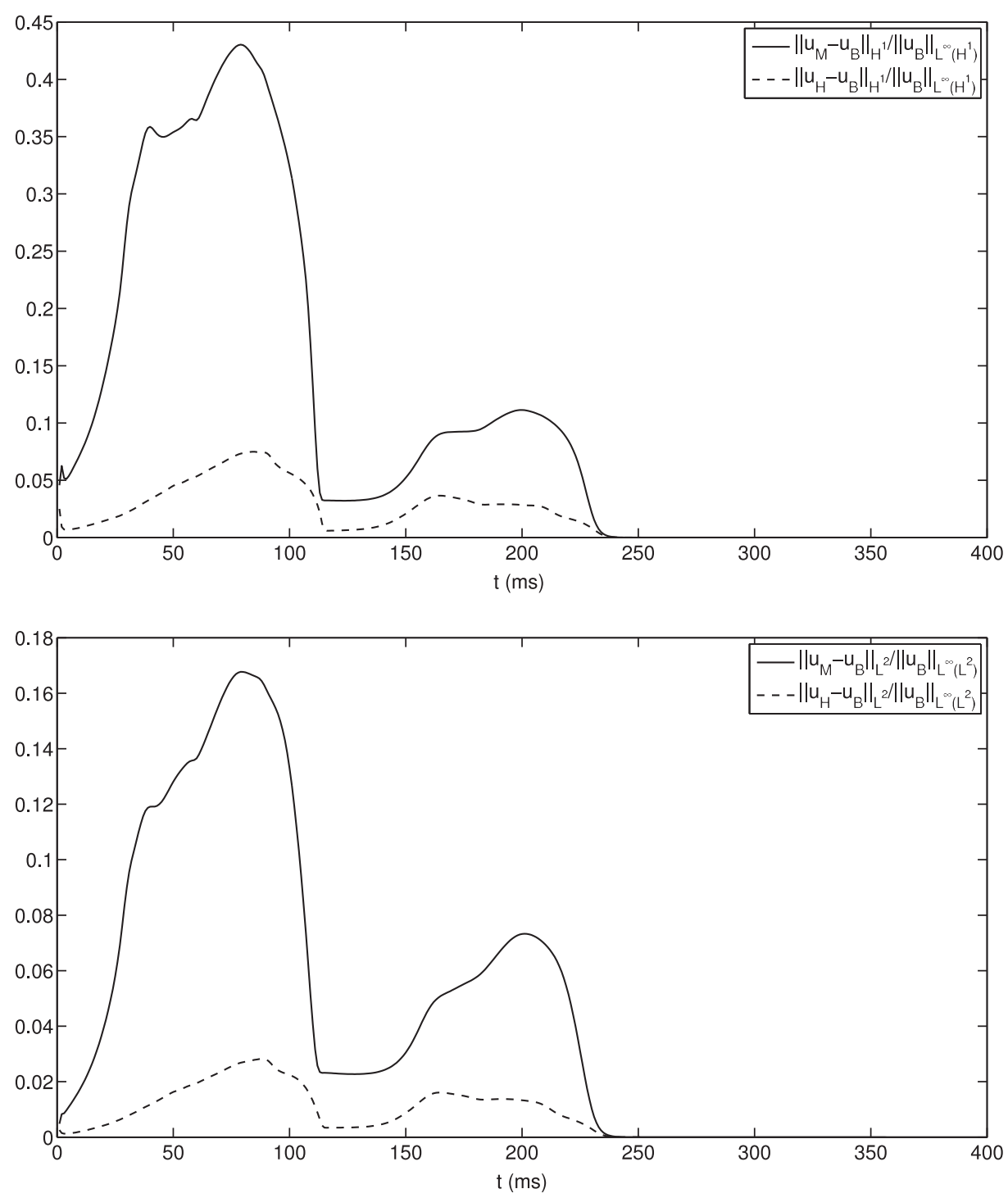

Fig. 5. Relative $H^{1}(\Omega)$ norm (top) and $L^{2}(\Omega)$ norm (bottom) of $e_{u, M}$ and $e_{u, H}$ at each time step of the simulation. 
ers. The geometry, the space discretization and the fibers direction used are the same as in the previous test case. In this case the conductivity of the tissue is non-homogeneous, the extracellular conductivities being amplified, while the intracellular conductivities are reduced, as suggested in [10].

Since the adaptive algorithm is independent of the choice of the ionic model, we analyze the performance of this strategy choosing

\section{Table 2}

Condition number of the preconditioned Bidomain (2nd column) and Hybridomain (3nd) matrices. In the first column we report the number of nodes of the computational mesh used. The Hybridomain matrix corresponds to $t=15 \mathrm{~ms}$. From all the Extended Monodomain, Bidomain and Hybrid matrices the singularity has been removed by enforcing, in an algebraic way, the zero average of the extracellular potential.

\begin{tabular}{lll}
\hline \# Mesh nodes & $K\left(\mathbf{M}^{-1} \mathbf{B}\right)$ & $K\left(\mathbf{M}^{-1} \mathbf{H}\right)$ \\
\hline 6288 & $1.67 \mathrm{e}+02$ & $5.04 \mathrm{e}+01$ \\
12,437 & $8.13 \mathrm{e}+01$ & $5.47 \mathrm{e}+01$ \\
22,470 & $1.81 \mathrm{e}+02$ & $3.96 \mathrm{e}+01$ \\
52,953 & $7.39 \mathrm{e}+01$ & $6.04 \mathrm{e}+01$ \\
\hline
\end{tabular}

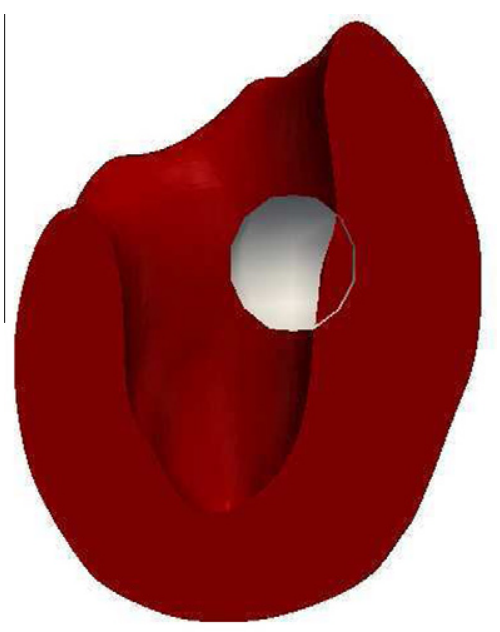

Fig. 6. Artificial scar on the wall of the SPECT reconstructed left ventricle geometry in use. only one of the two ionic models mentioned in Section 2, namely the Rogers-McCulloch one, whose parameters are specified in [7]. The time step is $\Delta t=0.5 \mathrm{~ms}$ and the simulations are carried out for $400 \mathrm{~ms}$. We point out that the transmembrane potential computed with Rogers-McCulloch model is shifted by $84 \mathrm{mV}$ with respect to the physiological one, setting $0 \mathrm{mV}$ as rest potential in place of the physiological $-84 \mathrm{mV}$.

\subsection{Test cases on a healthy real geometry}

The aim of the first set of simulations is to compare the choice of different thresholds. More precisely we set $\tau_{\text {eff }}=\tau^{2}$ ( $\tau$ being introduced in (28)), with respect to the effectivity index of the upper bound estimator defined at each time step as

$\theta^{u p}:=\frac{\eta_{\Omega_{M}}\left(u_{e, H}\right)}{\left\|e_{i, H}, e_{e, H}\right\|}$.

In Fig. 1 we show for every timestep the effectivity index (top) and the percentage of Bidomain elements over the total number of elements (bottom), for $\tau_{e f f}=20,40,80,120,160$. More precisely, since the rest transmembrane potential is zero, the error is dropping to zero in the last phase of the simulation and it forces the effectivity index to grow, even if the Hybridomain solution is identical to the Bidomain one. To filter this effect, in Fig. 1 we plot $\theta^{\text {up }}$ if $\left\|e_{i, H}, e_{e, H}\right\| \mid>10^{-3}$ and 0 otherwise.

In Fig. 2 we plot the average of the effectivity index with respect to the chosen threshold. The effectivity index is quite robust with respect to the choice of the threshold, ranging between 4.68 and 4.93. Moreover we identify $\tau_{\text {eff }}=80$ as the threshold value that gives the minimum effectivity index, and therefore the more effective adaptive strategy. In the subsequent simulations we set $\tau_{\text {eff }}=80$.

Fig. 3 shows the effectivity index of the lower bound estimator, defined as

$\theta^{\text {low }}=\frac{\zeta_{\text {opt }}\left(u_{e, H}\right)}{\left\|e_{i, H}, e_{e, H}\right\|}$.

Fig. 4 highlights (in red) the distribution in space of the active Bidomain elements (region $\Omega_{B}$ ) at three different time steps. Comparing the activation pattern with the Bidomain transmembrane potential pattern we stress that the adaptive strategy, based on
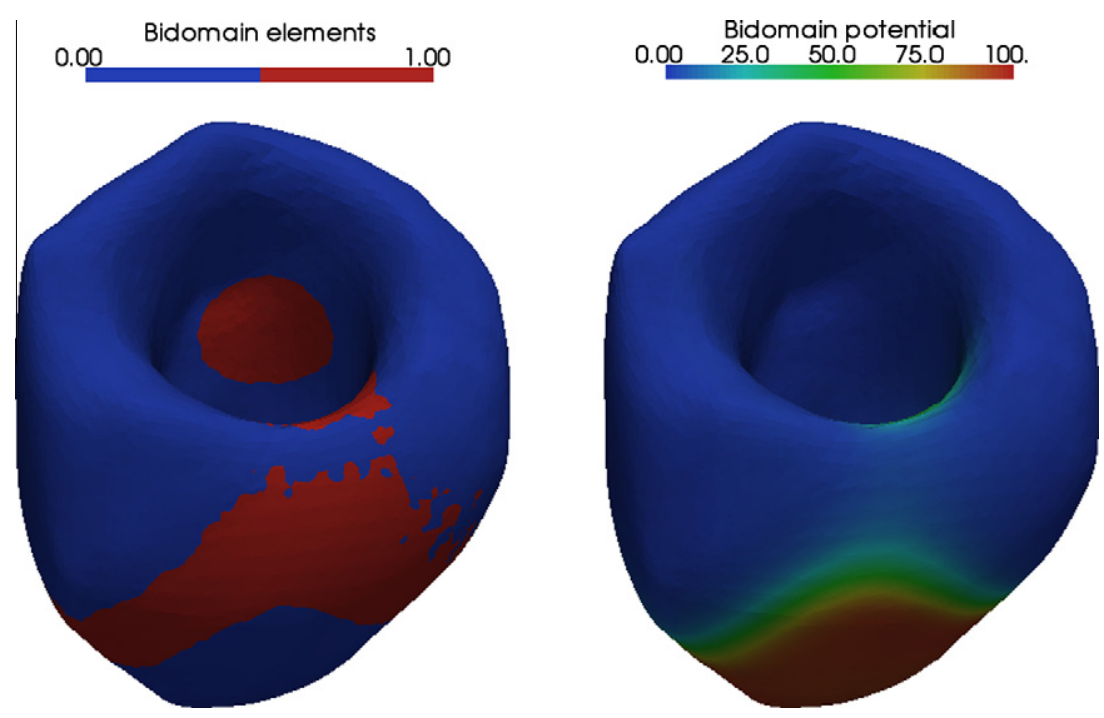

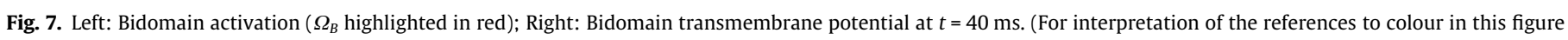
legend, the reader is referred to the web version of this article.) 

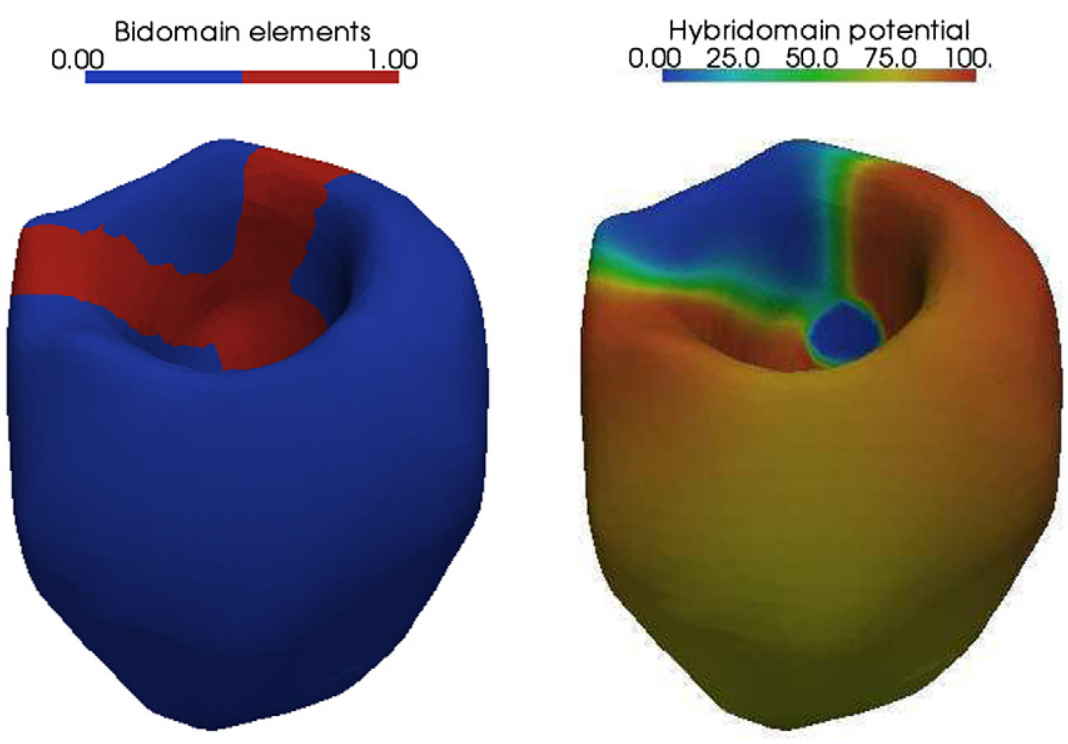

Monodomain potential

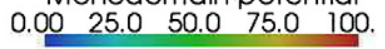

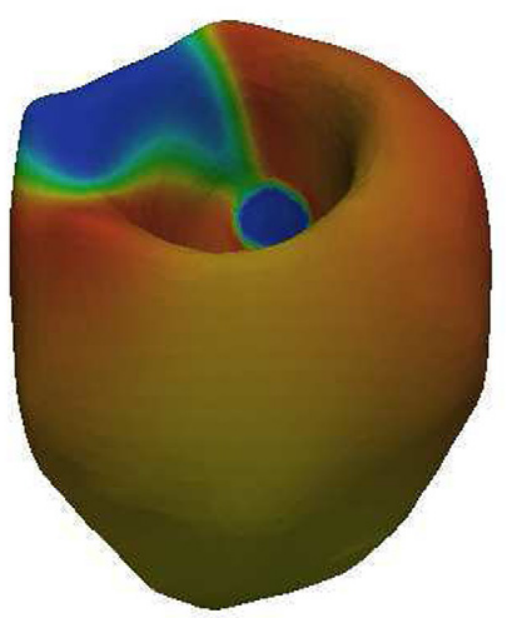

Bidomain potential

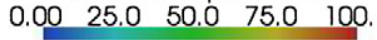

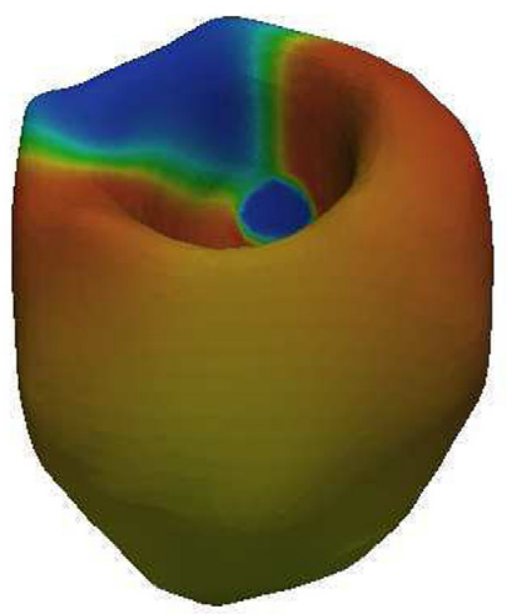

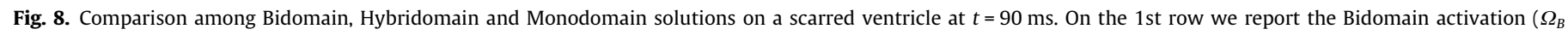

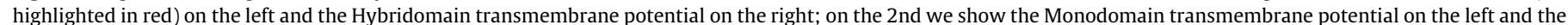

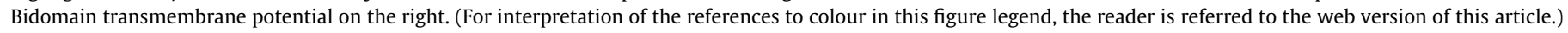

the estimator $\eta_{\Omega_{M}}\left(u_{e, H}\right)$, successfully activates the Bidomain model in the area involved by the propagating front. This confirms the reliability of the a priori error estimator.

Let us now calculate the effectiveness of the adaptive Hybridomain model both in reducing the error with respect to the simplified Monodomain model and in reducing the computational time with respect to the complete Bidomain model.

In Fig. 5 we compare the norm of the difference $e_{u, M}^{\text {tot }}$ with the norm of $e_{u, H}^{\text {tot }}$. Solving the Hybridomain in place of the Monodomain produces a solution much closer to the Bidomain one both in terms of the $H^{1}$ and $L^{2}$ errors.

In Table 1 we compare the computational effort required for solving the Bidomain system and the Hybridomain system. In particular we report the average CPU time (computed over all the time steps of the simulation) and the number of iterations required by the iterative algorithm GMRES to converge. As mentioned in Section 4, both systems are preconditioned with the Extended Monodomain, and in this test case the preconditioner system is solved with the Conjugate Gradient method, up to the fulfillment of an inner tolerance tol $=10^{-5}$. In the first row of the table we report the gain in iteration count

$g_{\text {it }}=100 \frac{\text { (\# Bidomain iterations })-(\text { Hybridomain iterations })}{\text { (\# Bidomain iterations })}$.

In the second row we show the gain in CPU time for the solution of each system in the time advancing scheme, computed as

$g_{\text {time }}=100 \frac{\text { (CPU Bidomain })-(\text { CPU Hybridomain })}{\text { (CPU Bidomain })}$.

When considering that solving the Hybridomain system requires to reassemble the upper right block of the matrix, the gain is defined as

$$
\begin{aligned}
g_{\text {time, net }}= & 100 \\
& \times \frac{(\text { CPU Bidomain })-(\text { CPU Hybridomain }+ \text { CPU assembling })}{(\text { CPU Bidomain })} .
\end{aligned}
$$


This is reported in the last row of the table. The adaptive strategy we are proposing, combined with the model preconditioning proposed in [12], allows therefore to save more than 30\% of CPU time with respect to solving the Bidomain and with an average error per time step (with respect to the Bidomain model) smaller than $2.9 \%$, compared to the $16 \%$ average error of the Monodomain solver.

Remark. We point out that if we use a coarser inner tolerance for CG method when solving the preconditioner system, as suggested in [12] (Flexible GMRES solver), the computational time required to solve both the Hybridomain and the Bidomain decreases and the difference between them becomes less evident. In particular in this test case $g_{\text {it }}=12.5 \%, g_{\text {time }}=10.9 \%$ and $g_{\text {time,net }}=6.2 \%$. Effectiveness of adaptivity is less evident in this case. More sophisticated coupling strategies that could avoid to resort to the Extended Monodomain model will allow a more relevant computing time reduction and will be investigated as a future development of the present work.

Condition number. Let $\mathbf{B}, \mathbf{M}$ and $\mathbf{H}$ be the matrices obtained after the discretization (in time and space) of the Bidomain, (Extended) Monodomain and Hybridomain models at a given time step. We analyze the condition number of the matrices $\mathbf{M}^{-1} \mathbf{B}$ and $\mathbf{M}^{-1} \mathbf{H}$ obtained by preconditioning the Bidomain system and the Hybridomain system with the Extended Monodomain matrix. Different computational meshes are considered. We report in Table 2 the condition number associated. For all the mesh sizes tested, the action of $\mathbf{M}$ as preconditioner is remarkable and the condition number of the Hybridomain preconditioned matrix is consistently smaller.

\subsection{Test cases on a real geometry with artificial scar}

In this test case we impose an artificial scar on the ventricle wall tissue, on the intersection between the previously described SPECT geometry and a sphere centered in $(0.8,-0.3,0) \mathrm{cm}$ and with $1 \mathrm{~cm}$ radius, as shown in Fig. 6 . For this test case we run a Bidomain, a Hybridomain and a Monodomain simulation and we compare the transmembrane potential patterns we obtain. In Fig. 7 we can see that the modeling error estimator activates the Bidomain model in the scar region, even if the propagating front is far from it. This behaviour could be useful if the scar region needs to be studied and analyzed in a more accurate way than the rest of the cardiac tissue, during the whole heart beat.

It is also evident from Fig. 8 that the propagating front predicted by the Hybridomain near the scar is more similar to the Bidomain front than the Monodomain pattern.

\section{Conclusion}

In this paper we have introduced a model adaptivity strategy for coupling Bidomain and Monodomain models in electrocardiology, in the form of a hybrid system, called Hybridomain. In this model, we couple a non symmetric formulation of the Bidomain system and an extended version of the Monodomain one, so that the Hybridomain model is obtained just switching on or off a term locally (i.e. elementwise in the finite element discretization). The region where we activate the "Bidomain term" is selected using a modeling error estimator introduced here. Numerical results testify the effectivity of the estimator and of the adaptive approach. There are some limitations that prevent this model adaptive solver to be more effective. In particular, the need of resorting to an Extended Monodomain formulation makes the reduced model still more expensive than the pure Monodomain problem. Coupling between Bidomain and pure Monodomain problems is however non trivial, for the different nature of the two problems and a specific devising of interface conditions is currently under investigation. On the other hand, the Hybridomain model presented here is easy to implement once a Bidomain non symmetric solver is available and provides a reliable image of the critical regions of potential propagation in the heart, both in the healthy and pathological cases.

\section{Acknowledgements}

F. Nobile has been partially supported by the Italian Project PRIN 2007 "Modellistica numerica per il calcolo scientifico ed applicazioni avanzate" and the European Research Council Advanced Grant "Mathcard, Mathematical Modelling and Simulation of the Cardiovascular System", Project ERC-2008-AdG 227058. The authors thank Dr. L. Gerardo-Giorda and Dr. M. Perego (Emory University) for many fruitful discussions in preparing this paper and Prof. E.V. Garcia for the SPECT images of left ventricles.

\section{References}

[1] N. Ajmone Marsan, M. Henneman, J. Chen, C. Ypenburg, P. Dibbets, S. Ghio, G. Bleeker, M. Stokkel, E.E. van der Wall, L. Tavazzi, E. Garcia, J. Bax, Left ventricular dyssyncrony assessed by two three-dimensional imaging modalities: phase analysis of gated myocardial perfusion SPECT and tri-plane tissue Doppler imaging, Eur. J. Nucl. Med. Mol. Imaging 35 (2008) 166-173.

[2] Y. Bourgault, Y. Coudiere, C. Pierre, Existence and uniqueness of the solution for the bidomain model used in cardiac electrophysiology, Nonlinear Anal.: Real World Appl. 10 (1) (2009) 458-482.

[3] M. Braack, A. Ern, A posteriori control of modeling errors and discretization errors, Multiscale Model. Simul. 1 (2) (2003) 221-238 (electronic).

[4] J. Chen, E.V. Garcia, R. Folks, C. Cooke, T. Faber, E. Tauxe, A. Iskandrian, Onset of left ventricular mechanical contraction as determined by phase analysis of ECG-gated myocardial perfusion SPECT imaging: development of a diagnostic tool for assessment of cardiac mechanical dyssyncrony, J. Nucl. Cardiol. 12 (2005) 687-695.

[5] J. Clements, J. Nenonen, P. Li, M. Horacek, Activation dynamics in anisotropic cardiac tissue via decoupling, Ann. Biomed. Engrg. 32 (2004) 984-990.

[6] P. Colli Franzone, L. Guerri, M. Pennacchio, B. Taccardi, Spread of excitation in 3-d models of the anisotropic cardiac tissue. III: Effects of ventricular geometry and fiber structure on the potential distribution, Math. Biosci. 151 (1998) 5198.

[7] P. Colli Franzone, L.F. Pavarino, A parallel solver for reaction-diffusion systems in computational electrocardiology, Math. Models Methods Appl. Sci. 14 (2004) 883-911.

[8] P. Colli Franzone, L. Pavarino, G. Savaré, Computational electrocardiology: mathematical and numerical modeling in complex systems, in: A. Quarteroni, L. Formaggia, A. Veneziani (Eds.), Biomedicine, Springer, Milan, 2006.

[9] P. Colli Franzone, G. Savaré, Degenerate evolution systems modeling the cardiac electric field at micro and macroscopic level, in: A. Lorenzi, B. Ruffa (Eds.), Evolution Equations Semigroups and Functional Analysis, 2002, pp. 218-240.

[10] D. Farina, O. Dossel, Model-based approach to the localization of infarction, Comput. Cardiol. (2007) 173-176.

[11] R. FitzHugh, Impulses and physiological states in theoretical models of nerve membrane, Biophys. J. 1 (1961) 445-466.

[12] L. Gerardo-Giorda, L. Mirabella, F. Nobile, M. Perego, A. Veneziani, A modelbased block-triangular preconditioner for the bidomain system in electrocardiology, J. Comput. Phys. 228 (2009) 3625-3639.

[13] C.S. Henriquez, A.L. Muzikant, C.K. Smoak, Anisotropy, fiber curvature, and bath loading effects on activation in thin and thick cardiac tissue preparations: simulations in a three-dimensional bidomain model, J. Cardiovasc. Electrophysiol. 7 (5) (1996) 424-444.

[14] J.P. Keener, Direct activation and defibrillation of cardiac tissue, J. Theor. Biol. 178 (1996) 313-324.

[15] L. Luo, Y. Rudy, A model of the ventricular cardiac action potential: depolarization, repolarization and their interaction, Circ. Res. 68 (1991).

[16] B.F. Nielsen, T.S. Ruud, G.T. Lines, A. Tveito, Optimal monodomain approximation of the bidomain equations, Appl. Math. Comput. 184 (2007) 276-290.

[17] J.T. Oden, S. Prudhomme, Estimation of modeling error in computational mechanics, J. Comput. Phys. 182 (2) (2002) 496-515.

[18] M. Pennacchio, V. Simoncini, Efficient algebraic solution of reaction-diffusion systems for the cardiac excitation process, J. Comput. Appl. Math. 145 (2002) 49-70.

[19] S. Perotto, Adaptive modeling for free-surface flows, M2AN Math. Model. Numer. Anal. 40 (3) (2006) 469-499.

[20] M. Potse, B. Dub, A. Vinet, Comparison of monodomain and bidomain models for whole-heart propagation studies, in: The 33rd International Congress on Electrocardiology, Cologne, June 2006. 
[21] S. Prudhomme, H. Ben Dhia, P.T. Bauman, N. Elkhodja, J.T. Oden, Computational analysis of modeling error for the coupling of particle and continuum models by the Arlequin method, Comput. Methods Appl. Mech. Engrg. 197 (41-42) (2008) 3399-3409.

[22] A. Pullan, M. Buist, L. Cheng, Mathematical Modelling the Electrical Activity of the Heart, World Scientific, Singapore, 2005.

[23] J. Rogers, A. McCulloch, A collocation-Galerkin finite element model of cardiac action potential propagation, IEEE Trans. Biomed. Engrg. 41 (1994) 743-757.

[24] Y. Rudy, J. Silva, Computational biology in the study of cardiac ion channels and cell electrophysiology, Quarter. Rev. Biophys. 39 (2006) 57-116.

[25] Y. Saad, Iterative Methods for Sparse Linear Systems, PWS, Boston, 1996.

[26] F.B. Sachse, Computational Cardiology, Springer, Berlin, 2004.

[27] S. Scacchi, L.F. Pavarino, Multilevel Schwarz and multigrid preconditioners for the bidomain system, in: Domain Decomposition Methods in Science and Engineering (Proceedings of the DD17 Conference), Springer-Verlag, 2007, pp. 631-638.
[28] K.S. Vemaganti, J.T. Oden, Estimation of local modeling error and goal-oriented adaptive modeling of heterogeneous materials. II. A computational environment for adaptive modeling of heterogeneous elastic solids, Comput. Methods Appl. Mech. Engrg. 190 (46-47) (2001) 6089-6124.

[29] M. Veneroni, Reaction-diffusion systems for the microscopic cellular model of the cardiac electric field, Math. Method Appl. Sci. 29 (2006) 1631-1661.

[30] E.J. Vigmond, F. Aguel, N.A. Trayanova, Computational techniques for solving the bidomain equations in three dimensions, IEEE Trans. Biomed. Eng. 49 (2002) 1260-1269.

[31] R. Weber dos Santos, G. Planck, S. Bauer, E. Vigmond, Parallel multigrid preconditioner for the cardiac bidomain model, IEEE Trans. Biomed. Engrg. 51 (2004) 1960-1968.

[32] R.L. Winslow, J.J. Rice, S. Jafri, E. Marban, B. O’Rourke, Mechanisms of altered excitation-contraction coupling in canine tachycardia-induced heart failure. II: Model studies, Circ. Res. 84 (1999) 571-586. 